Intraoperative US for renal tumors can also be used also to identify satellite lesions, assess for peritumoral vascularity, determine renal vein invasion and also to demonstrate the cranial extension of tumor thrombus within the inferior vena cava. Radiofrequency ablation and cryoablation can also be monitored by intraoperative ultrasound.

Dr. Adilson Prando

Head, Department of Radiology and Diagnostic Imaging, Vera Cruz Hospital

Campinas, São Paulo, Brazil

E-mail: adilson.prando@gmail.com

\title{
PATHOLOGY
}

doi: 10.1590/S1677-55382011000200021

\section{Active surveillance program for prostate cancer: an update of the Johns Hopkins experience} Tosoian JJ, Trock BJ, Landis P, Feng Z, Epstein JI, Partin AW, Walsh PC, Carter HB

The Johns Hopkins University School of Medicine, The James Buchanan Brady Urological Institute, and Johns Hopkins Hospital, Baltimore, MD

J Clin Oncol. 2011; 4. [Epub ahead of print]

Purpose: We assessed outcomes of men with prostate cancer enrolled in active surveillance.

Patients and Methods: Since 1995, a total of 769 men diagnosed with prostate cancer have been followed prospectively (median follow-up, 2.7 years; range, 0.01 to 15.0 years) on active surveillance. Enrollment criteria were for very-low-risk cancers, defined by clinical stage (T1c), prostate-specific antigen density $<0.15$ $\mathrm{ng} / \mathrm{mL}$, and prostate biopsy findings (Gleason score $\leq 6$, two or fewer cores with cancer, and $\leq 50 \%$ cancer involvement of any core). Curative intervention was recommended on disease reclassification on the basis of biopsy criteria. The primary outcome was survival free of intervention, and secondary outcomes were rates of disease reclassification and exit from the program. Outcomes were compared between men who did and did not meet very-low-risk criteria.

Results The median survival free of intervention was 6.5 years (range, 0.0 to 15.0 years) after diagnosis, and the proportions of men remaining free of intervention after 2,5 , and 10 years of follow-up were $81 \%, 59 \%$, and $41 \%$, respectively. Overall, 255 men (33.2\%) underwent intervention at a median of 2.2 years (range, 0.6 to 10.2 years) after diagnosis; 188 men (73.7\%) underwent intervention on the basis of disease reclassification on biopsy. The proportions of men who underwent curative intervention $(\mathrm{P}=0.026)$ or had biopsy reclassification $(\mathrm{P}<0.001)$ were significantly lower in men who met enrollment criteria than in those who did not. There were no prostate cancer deaths.

Conclusion: For carefully selected men, active surveillance with curative intent appears to be a safe alternative to immediate intervention. Limiting surveillance to very-low-risk patients may reduce the frequency of adverse outcomes.

\section{Editorial Comment}

The authors studied the outcomes of men with prostate cancer enrolled in active surveillance comparing patients who did and did not meet very-low-risk criteria. Very-low-risk was defined according to the contemporary analysis of Bastian et al. for Epstein's criteria for insignificant cancer on needle biopsy: clinical stage 
T1c, prostate-specific antigen density $<0.15 \mathrm{ng} / \mathrm{mL}$, Gleason score $\leq 6$, two or fewer cores with cancer, and $\leq$ $50 \%$ cancer involvement of any core.

During the follow-up period the proportions of men who underwent curative intervention $(p=0.026)$ or had biopsy reclassification (more than 2 cores, Gleason score $>6$, or $>50 \%$ cancer involvement of any core) $(\mathrm{p}<0.001)$ were significantly lower in men who met very-low-risk criteria. There were also no prostate cancer deaths in this cohort of patients.

The authors conclude that for carefully selected men, active surveillance limited to patients with verylow-risk cancers according to Epstein's criteria for insignificant cancer may significantly reduce the frequency of adverse outcomes.

\title{
Reference
}

1. Bastian PJ, Mangold LA, Epstein JI, Partin AW: Characteristics of insignificant clinical T1c prostate tumors. A contemporary analysis. Cancer. 2004; 101: 2001-5.

\author{
Dr. Athanase Billis \\ Full-Professor of Pathology \\ State University of Campinas, Unicamp \\ Campinas, São Paulo, Brazil \\ E-mail: athanase@fcm.unicamp.br
}

doi: 10.1590/S1677-55382011000200022

\section{Testicular Vasculitis: A Series of 19 Cases}

Brimo F, Lachapelle J, Epstein JI

Department of Pathology, McGill University Health Center, Montreal, Canada

Urology. 2011; 16. [Epub ahead of print]

Objectives: Because of limited reported cases, it is unknown how often testicular vasculitis represents isolated or systemic disease.

Methods: We report 19 cases (15 consultation; 4 in-house cases) of localized testicular infarction with associated vasculitis spanning 24 years.

Results: All were orchiectomy specimens; detailed clinical information was available in 16 cases. Mean age was 38 years. Clinical presentation was testicular pain in 13 and mass in 3 patients. Preoperative impression was testicular cancer in 13 cases. In all cases, localized testicular infarction associated with vasculitis was present and in none was tumor identified. Most cases $(n=14)$ showed polyarteritis nodosa $(P A N)$-like features with transmural necrotizing inflammation of small-medium arteries. In 4 cases, vasculitis was granulomatous (2 necrotizing; 2 non-necrotizing) and in 1 case was lymphocytic. An infectious etiology was excluded clinically and by special stains. Four patients were subsequently confirmed with systemic vaculitis: one with PAN, one with Wegener vasculitis, one with vasculitis not otherwise specified and one with subclinical systemic vasculitis. Two of those 4 patients had testicular PAN-like vasculitis and 2 had granulomatous vasculitis.

Conclusions: Testicular vasculitis can cause localized infarction that clinically mimics cancer. Although testicular vasculitis is an isolated finding in most patients an associated systemic vasculitis is not a rare event (4/16, or $25 \%$ ), especially if the vasculitis is granulomatous (50\% in this series). All patients should be clinically investigated for systemic disease. 